\title{
Corneal Ulcer
}

National Cancer Institute

\section{Source}

National Cancer Institute. Corneal Ulcer. NCI Thesaurus. Code C50515.

Area of epithelial tissue loss from corneal surface; associated with inflammatory cells in the cornea and anterior chamber. 